\title{
Formalin-Fixed Paraffin-Embedded
}

National Cancer Institute

\section{Source}

National Cancer Institute. Formalin-Fixed Paraffin-Embedded. NCI Thesaurus. Code C143028.

Refers to a process where a sample is preserved with formalin and then embedded into a paraffin block for sectioning. 